\title{
Influence of substrate charge on electron transport in narrow conducting channel
}

\author{
Yu.Z. Kovdrya, V.A. Nikolaenko, and A.V. Smorodin \\ B.I.Verkin Institute for Low Temperature Physics and Engineering, National \\ Academy of Sciences of Ukraine, 47 Lenin avenue, 61103 Kharkov, Ukraine
}

\begin{abstract}
The electron transport in inhomogenous quasi-one-dimensional conducting channels on the liquid helium surface are studied in the temperature range $0.6-1.5 \mathrm{~K}$. Inhomogeneities are created by charging the substrate on which the conducting channels are formed. It has been established that the electron conductivity practically does not depend on temperature at some substrate charge. The results obtained are explained by localization of carriers and creation of electron polarons.

PACS numbers: 67.40.Jd, 73.20-r.
\end{abstract}

\section{INTRODUCTION}

The investigations of electron transport in the system with restricted geometry are of great interest ${ }^{1}$. It was proved to be possible to realize a quasi-one-dimensional (Q1D) charge system with using surface electrons on liquid helium $(\mathrm{SE})^{2}$. It has been observed that character of the transport in conducting Q1D channels essentially different in the channels of different width and quality of the substrate surface on which the conducting channels are formed. For a clean homogenous substrate and narrow conducting channels, the electron mobility is determined by interaction of carriers with helium atoms in vapor and ripplons 3 and is described well by the theory. ${ }^{[5}$ As the width of the conducting channel increases, an anomalous charge transport is observed ${ }^{6}[7]$; the mobility first increases with decreasing temperature $T$, and then at $T<0.8 \mathrm{~K}$ starts to decrease. The quality of the substrates influences on the character of the electron transport. As it was suggested, this effect is connected with appearance of potential wells 


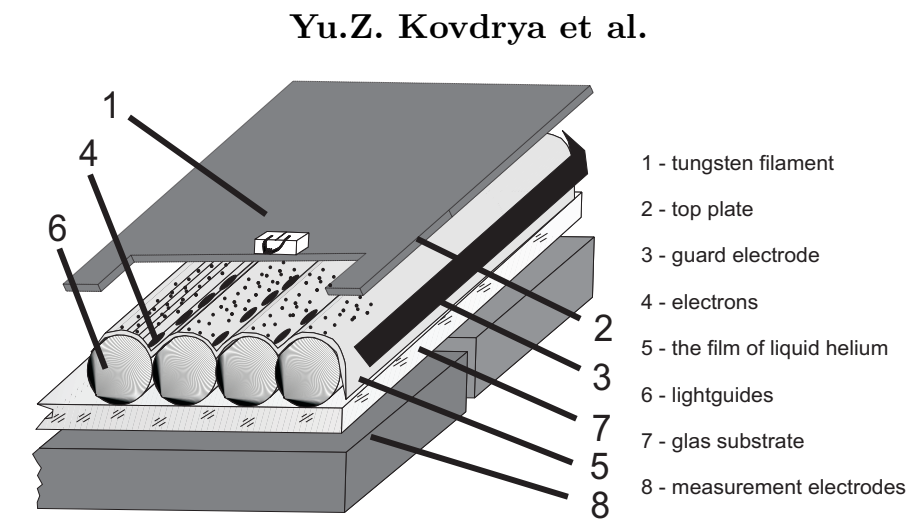

Fig. 1. Schematic of the experimental cell

due inhomogeneities on the surface of the substrates. For creation of potential wells it is convenient to use electron charge deposited on the surface of a substrate. In this case it is possible to change by a controlled way the density of the potential wells. Preliminary data on the investigation of the electron mobility in Q1D channels with charged surface have been obtained in Ref. 7; in this work the results of detailed investigations of the influence of the electron substrate charge on the carrier conductance in Q1D channels over liquid helium are presented.

\section{EXPERIMENTAL}

For the experimental investigation of conductivity, the cell consisting of the top and bottom metal plates between which a profiled dielectric substrate is placed (Fig. 1). The bottom plate of the cell represents a coplanar system of measuring electrodes consisting of two equal parts by size $6 \times 13.5 \mathrm{~mm}^{2}$ and with a gap between them $a \simeq 10$ microns. The small value of the gap practically excludes a nonuniformity of electric field in a plane of the substrate which is proportional to $\exp (-b / a)$, where $b$ is distance from the lower plate to the electron channels.

The structure of the substrate and technique of measurement of conductivity were similar described in Ref. 4. A signal of frequency $20 \mathrm{kHz}$ from a generator was applied to the one of the electrodes, the signal passing through the experimental cell was taken off from the another electrode. The substrate represents a 50 lightguides of diameter 240 microns and length 12 $\mathrm{mm}$ located on a glass plate of the size $12 \times 13,5 \times 0,2 \mathrm{~mm}^{3}$. Liquid helium covers the substrate realizing "deep" liquid channels with some surface curvature between the lightguids. The tops of the lightguides are covered with the liquid film of the thickness of about $300 \AA$. The shift of amplitude of $0^{0}$ and $90^{\circ}$ components of an electrical signal, passing through the cell 


\section{Influence of substrate charge on electron transport in narrow conducting channel}

with electrons, were measured; this allowed to determine the real $G_{r}$ and imaginary $G_{i}$ parts of the conductance of the cell.

Theoretical calculation of $G_{r}$ and $G_{i}$ with taking into account the cell geometry are resulted in Ref. 4 . For the values of $G_{r}$ and $G_{i}$ the following expression has been obtained:

$$
\begin{gathered}
G_{r}=n_{s} e^{2} \cdot \sum_{n, l=1}^{\infty} \frac{\Lambda_{n, l} e \omega^{2} \chi_{1} \lambda}{\left(m \omega_{q}^{2}-e \omega^{2} \chi_{2} \lambda\right)^{2}+\left(e \omega^{2} \chi_{1} \lambda\right)^{2}}, \\
G_{i}=n_{s} e^{2} \cdot \sum_{n, l=1}^{\infty} \frac{\Lambda_{n, l}\left(m \omega_{q}^{2}-e \omega \chi_{2} \lambda\right) \omega}{\left(m \omega_{q}^{2}-e \omega \chi_{2} \lambda\right)^{2}+\left(e \omega^{2} \chi_{1} \lambda\right)^{2}}+g_{0} .
\end{gathered}
$$

Here the value $g_{0}$ characterizes conductance of the cell without electrons, value $\chi_{1}$ and $\chi_{2}$ are real and imaginary components of resistivity of the channels on one electron, $n_{s}$ is average density electrons in the cell, $\omega_{q}$ is frequency of the plasma oscillations propagating in system of parallel channels. The parameters $\Lambda_{n, l}$ and $\lambda$ in expressions (1) and (2) are determined by the cell geometry. Estimations show that the main contribution to value $G_{r}$ and $G_{i}$ is given by the first term. Expressions (1), (2) allowed to determine conductivity $\sigma$ of the channels and the value $a: a=\frac{m \omega_{q}^{2}-e \omega \chi_{2} \lambda}{n_{s}}$. The value $a$ is determined by frequency of plasma oscillations and the imaginary part of resistivity of the channels. For free electrons $m \omega_{q}^{2}>>e \omega \chi_{2} \lambda$, and the value $a=\frac{m \omega_{q}^{2}}{n_{s}}$. Since $\omega_{q}^{2}$ is proportional to $n_{s}$ and inversely to $m$ (Ref. 4) the value $a$ in this case depends only on the cell geometry.

\section{DISCUSSION}

The results obtained are presented in Figs. 2, 3. A typical dependence of the conductivity of the channels $\sigma$ on a pressing potential $V_{\perp}$ is shown in Fig. 2. The curve has been obtained in the condition of saturated charge. It is seen, that the signal appears not with zero but from some value $V_{\perp c r}$. The value $V_{\perp c r}$ since which the signal appears is determined by density of electrons localized on the helium film and on the substrate surface. In Fig. 3a temperature dependence of the conductivity of the channels for the electron density $\sim 4 \cdot 10^{12} \mathrm{~m}^{-2}$ is presented. It is seen, that $\sigma$ at first slightly increases with decreasing temperature. At $T \approx 0.9 \mathrm{~K}$ a step changing of the value $\sigma$ approximately $10 \%$ is observed. Unfortunately, the nature of such a decreasing $\sigma$ is not clear at the moment, and the additional experiments for the explanation of this effect are needed. The week temperature dependence of $\sigma$ allows to conclude that the helium atoms in vapor and ripplons 
Yu.Z. Kovdrya et al.

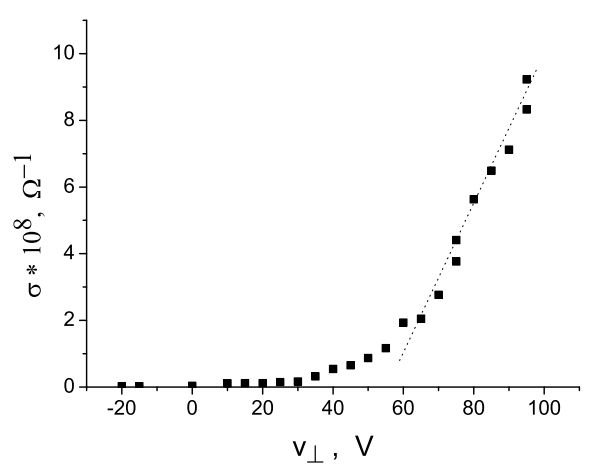

Fig. 2. A typical dependence of the conductivity of the channels $\sigma$ on a pressing potential $V_{\perp}$.

practically don't influence on the conductivity of the channels.

In the region of temperatures $0.6-1.4 \mathrm{~K}$ the value $a$ practically does not depend on temperature (Fig. 3b). As it follows from Ref. 4, the theoretical value of $a$ depends only on the cell geometry and must be independent on temperature. The experimentally found values of $a$ is approximately two orders more than the theoretical values of $a$ obtained for the ideally conducting channels, and equals $\sim 2.4 \cdot 10^{-26} \mathrm{~kg} \cdot \mathrm{m}^{2} / \mathrm{s}^{2}$. The plasma frequency, which has been calculated with using the results of Ref. 4 , is $\sim 5.1 \cdot 10^{8} \mathrm{~s}^{-1}$. The value $\omega_{q}$, calculated from the experimental value $a$, is $\omega_{q}=2.3 \cdot 10^{9}$ $s^{-1}$. It has been assumed in Refs. 3, 7, that increase of the plasma frequency appears when localization of particles in channels takes place. In this case, an additional, so-called, "optical mode" appears; the value $\omega_{q}$ thus can be presented as $\omega_{q}=\sqrt{\Omega_{0}^{2}+\omega_{p}^{2}}$, where $\omega_{p}$ is plasma frequency of ideal conducting channels. $\frac{3}{8}$ Estimations with using of the experimentally found value $a$ give for the $\Omega_{0}$ the value $\Omega_{0}=2.3 \cdot 10^{9} \mathrm{~s}^{-1}$. We can suppose, therefore, that electrons in quasi-one-dimensional channels in presence of a charge on the substrate are localized. Characteristic value of the localization length of a particle is $l_{l o c} \approx\left(\frac{\hbar}{m \Omega_{0}}\right)^{1 / 2}=2.2 \cdot 10^{-5} \mathrm{~cm}$. This value practically coincides with average distance between electrons in the channels $a_{0} \approx n_{s}^{-1 / 2}=2.4 \cdot 10^{-5} \mathrm{~cm}$.

One can supposed that such localization of particles can lead to formation electron polarons: microscopic dimples on the liquid helium surface with the effective mass noticeably exceeding the mass of free electrons. The value $n_{s}$ allows to estimate the mobility of electron polarons in conducting channels, which is equal $\mu \approx 0.125 \mathrm{~m}^{2} / V \cdot s$. It is much less than the theoretical value of the electron mobility in Q1D channels which is $\mu=12$ 


\section{Influence of substrate charge on electron transport in narrow conducting channel}
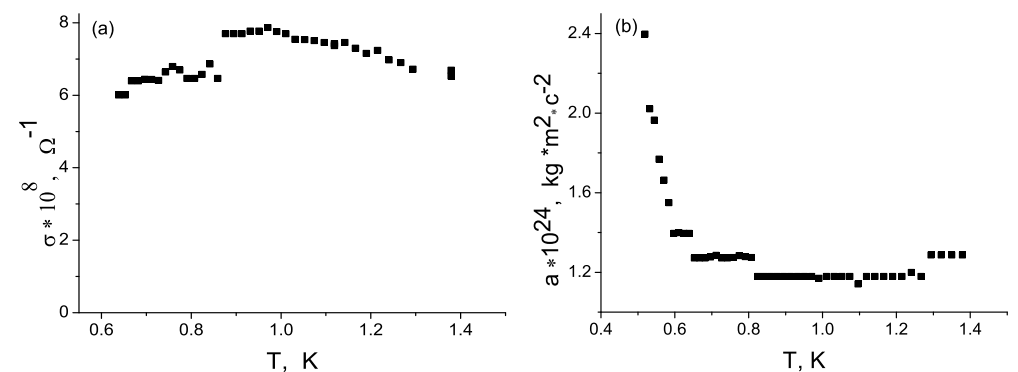

Fig. 3. The conductivity of the channels $\sigma$ (a) and the value $a$ (b) as $a$ function of temperature.

$m^{2} / V \cdot s$ at $T=1.4 \mathrm{~K}$. In the conducting Q1D channels above liquid helium, with enough good homogeneity, a strong temperature dependence of the electron mobility is observed ${ }^{4}$. In this connection, the week temperature dependence of $\sigma$, observed in the Q1D channels at presence of a charge on the substrate, is interesting. One of the possible explanations of this effect consists of that due to the large number of the potential wells formed by the immobile electrons, the carrier transport is realized by tunneling of particles from one potential well to another. The diffusion of particles in this case is determined by the expression $D \approx \nu_{0} \cdot a_{0}^{2} \cdot \exp \left[\int_{a}^{b} \sqrt{2 m \Delta V} d x\right]$, where $\nu_{0}=\frac{\Omega_{0}}{2 \pi}$ is frequency of oscillations of an electron in a potential well, $\Delta V$ is height of the potential well. The value $a_{0}$ set the characteristic scale of variations of the potential relief, it is determined by average distance between the electrons localized on a thin helium film and on the dielectric substrate. The appreciations, however, show that, for an explanation of observable values of the mobility, it is required to assume that $\Delta V=0.2 \mathrm{~K}$. It is too small value for realization of the tunnelling mechanism. More probable explanation of observed features of the carrier transport, is scattering electron polarons by variations of the potential, created both localized immobile electrons, and inhomogeneities of channels.

The electron transport in quasi-one-dimensional channels on the liquid helium surface at presence of inhomogeneities on a substrate was considered in Ref. 9. It has been shown that the mobility of carriers limited by scattering of electrons by defects, can be presented as

$$
\mu_{d} \approx \frac{e}{m \nu_{d}}\left(\frac{k T}{\hbar \omega_{0}}\right)^{1 / 2}
$$

Here $\nu_{d}$ is frequency of collisions electrons with surface defects of the sub- 


\section{Yu.Z. Kovdrya et al.}

strate, $k$ is Boltzman constant, $\hbar$ is Plank's constant, $\omega_{0}$ is frequency of oscillations of an electrons due to the movement of an electron across the channel. As defects the roughness of the substrate on which the conductivity channels are formed were considered. It was also supposed that a layer of liquid helium in a channel thin enough. Besides, at calculation the condition $k T<<\hbar \omega_{0}$ was used. It has been established that the value $\nu_{d}$ does not depend on temperature and consequently temperature dependence of $\mu$ is enough weak: $\mu \sim T^{1 / 2}$. In conditions of our experiment $\hbar \omega_{0}=0.2 \mathrm{~K}$, so the requirement $k T<<\hbar \omega_{0}$ does not take place. Beside this scattering of the electrons on variations of the random potential, probably, has Coulomb character. Nevethelees, apparently, one can expect that weak temperature dependence of the mobility in this case should takes place. Unfortunately, now there is no theory of the electron transport under the scattering electrons by the random potential caused by the charge of the substrate.

Thus, in the present work the electron transport in the quasi-onedimensional channels is investigated at presence of a charge on the substrate on which the channels are formed. It is shown that the conductivity of the channels practically does not depend on temperature. It is made the assumption that localization of carriers in the non-uniform channels, apparently, is accompanied with formation of electron polarons. The effective mass of such a polaron essentially exceeds the free electron mass.

\section{REFERENCES}

1. V.B. Shikin, Yu.P. Monarcha, "Two-dimensional Charged Systems in the Helium" Nauka, Moscow, (1989).

2. Yu.Z. Kovdrya and V.A. Nikolaenko, Fiz. Nizk. Temp. 18,1278 (1992) [Low. Temp. Phys. 18, 894 (1992)].

3. Yu.Z. Kovdrya, V.A. Nikolaenko, H. Yayama, A. Tomokigo, O.I. Kirichek, and I.B. Berkutov, J. Low. Temp. Phys. 110, 191 (1998).

4. S.P. Gladchenko, V.A. Nikolaenko, Yu.Z. Kovdrya, and S.S. Sokolov, Fiz. Nizk. Temp. 27,3 (2001) [Low. Temp. Phys. 27, 1 (2001)].

5. S.S. Sokolov, Guo-Qiang Hai, and N. Studart, Phys. Rev. B 51, 5977 (1995).

6. P. Glasson, V. Dotsenko, P. Foozoni, M.J. Lee, W. Bailey, G. Papageorgiou, S.E. Anderson, and A. Kristensen, Phys. Rev. Lett. 87, 176801 (2001).

7. S.P.Gladchenko, Yu.Z.Kovdrya, and V.A.Nikolaenko, Fiz. Nizk. Temp. 29,1265 (2003) [Low. Temp. Phys. 29, 960 (2003)].

8. E.Y. Andrei, Phys. Rev. Lett. 52, 1449 (1984).

9. S.S. Sokolov and N. Studart, Phys. Rev. B 66, 075424 (2002). 